\title{
El ejercicio de la supervisión en los servicios de atención a familias ${ }^{12}$
}

Sonia Juliana Pérez Pérez

Magister en Musicoterapia Universidad Internacional Iberoamericana. Colombia

Correo electrónico: sonia.perez@unini.org

\section{Esperanza Pérez Jiménez \\ Socióloga Investigadora pensionada \\ Correo electrónico: perita07@hotmail.com}

\section{Beatriz Amanda Arévalo Carrascal}

Especialista en Gerencia de Recursos Humanos Pontificia Universidad Javeriana. Bogotá, Colombia

Correo electrónico: arevalob@javeriana.edu.co

Recibido: 22/04/2019

Evaluado: 28/05/2019

Aceptado: 27/06/2019

\section{Resumen}

El objetivo de este artículo es presentar una reflexión sobre las características de la práctica de la supervisión en los servicios psicosociales de atención a familias, teniendo claro que no se trata de un documento orientado necesariamente a la práctica clínica, pero que se nutre de ésta para hacer aportes a los equipos interdisciplinarios que acompañan procesos relacionados. La metodología empleada consistió en la revisión de los procedimientos, fases y escenarios de la supervisión, haciendo énfasis en la conversación como materia prima de esta práctica. Desde un abordaje sistémico y constructivista, se destaca el ejercicio de la supervisión como una obligación ética desde la óptica de la enorme responsabilidad que encarnan los procesos de intervención con familias, como también desde el necesario autocuidado de los profesionales y las instituciones.

\section{Palabras clave}

Atención a familias, enfoque sistémico, intervención psicosocial, supervisión.

12 Para citar este artículo: Pérez-Pérez, S. J., Pérez-Jiménez, E. \& Arévalo-Carrascal, B. A. (2020). El ejercicio de la supervisión en los servicios de atención a familias. Informes Psicológicos, 20(2), pp. 171-183 http://dx.doi. org/10.18566/infpsic.v20n2a12 


\title{
The exercise of supervision in family care services
}

\begin{abstract}
The objective of this article is to present a reflection on the characteristics of the practice of supervision in psychosocial care services for families. This is not a document necessarily oriented to clinical practice, but that it draws on it to make contributions to the interdisciplinary teams that accompany related processes. The methodology used consisted of reviewing the supervision procedures, phases and scenarios, emphasizing conversation as the raw material for this practice. From a systemic and constructivist approach, the exercise of supervision stands out as an ethical obligation from the point of view of the enormous responsibility that the processes of intervention with families embody, as well as from the necessary self-care of professionals and institutions.
\end{abstract}

Keywords

Family care, systemic approach, psychosocial intervention, supervision.

\section{O exercício da supervisão nos serviços de assistência familiar}

\section{Resumo}

0 objetivo deste artigo é apresentar uma reflexão sobre as características da prática da supervisão nos serviços de atenção psicossocial às famílias, deixando claro que este não é um documento necessariamente orientado para a prática clínica, mas que se baseia nela para fazer contribuições para as equipes interdisciplinares que acompanham os processos relacionados. A metodologia utilizada consistiu na revisão dos procedimentos, fases e cenários da supervisão, enfatizando a conversação como matéria-prima para essa prática. Sob uma abordagem sistêmica e construtivista, 0 exercício da supervisão se destaca como uma obrigação ética, sob a perspectiva da enorme responsabilidade que os processos de intervenção com as famílias incorporam, bem como do necessário autocuidado de profissionais e instituições.

\section{Palavras chave} Assistência familiar, abordagem sistêmica, intervenção psicossocial, supervisão. 


\section{ntroducción}

Los equipos interdisciplinares de los servicios de atención a familias suelen constituirse por diferentes profesionales sobre los que recae la responsabilidad de asesorar, promover y acompañar el cambio de los sistemas familiares para la consolidación de un mayor bienestar. Es cada vez más frecuente la práctica supervisada que permite el análisis ético y reflexivo de los casos que atienden, en la búsqueda de beneficios para todos los involucrados. Ciertamente, "la práctica de la supervisión y el asesoramiento de equipos de servicios sociales está haciendo aparición, aunque lentamente, en diferentes escenarios" (Puig, 2011, p.132), siendo pertinente desde la creciente comprensión de parte de las instituciones y profesionales involucrados acerca de las necesidades y potencialidades colaborativas de los equipos. En este orden de ideas, las siguientes reflexiones tienen sentido para el desarrollo de procesos de intervención que, al desarrollarse en el marco de relaciones de ayuda desde el trabajo social, la psicología, la pedagogía, la sociología, etcétera, buscan un efecto bien sea terapéutico o transformacional que puede fortalecerse con el aporte de la supervisión.

Se entiende que la supervisión, desde un enfoque sistémico y constructivista, es un ejercicio necesario que reconoce las propias limitaciones y los recursos de otros profesionales, e incorpora en la práctica del agente de intervención (el profesional que realiza procesos de intervención), la construcción colaborativa para la activación de recursos prácticos, para que sus aportes e intervenciones se complejicen, se amplíen, se enriquezcan e innoven, de forma que su quehacer con las familias logre configurar contextos de transformación de sus dilemas y de sus relaciones, desarrollados en permanente reflexividad para el potenciamiento del cambio.

\section{¿En qué consiste la supervisión?}

Quien realiza intervenciones con familias es integrante de diversos sistemas de relaciones significativas que involucran amigos, familiares y especialmente otros profesionales con los que comparte y confronta su práctica. En tales contextos, el agente de intervención reflexiona acerca de las familias y en palabras de Hernández-Córdoba (2007a), sobre:

sus temores, sus ambiciones, etc., pero también sobre la naturaleza, los objetivos y su rol en la relación terapéutica. Este trabajo de autorreferencia reiterado orienta al terapeuta frente a su consultante y le sirve como una vigilancia ética y técnica (p. 290).

La supervisión surge como espacio idóneo para esta orientación, desde el compartir que valida el conocimiento colaborativo a la vez que desafía el concepto de objetividad y experticia, por lo cual se plantea el reto de poner en tela de juicio la propia práctica y las verdades individuales, para enriquecerlas con la mirada de otros que hacen parte del equipo de profesionales, mientras que se entrelazan perspectivas propias de los diferentes sistemas involucrados. La supervisión se entiende, entonces, como un ejercicio reflexivo acerca de la propia práctica 
(Fruggeri, 2001), en cuyo proceso los propios constructos se pueden abrir a otros, como también dar a luz nuevas ideas y formas de reconocer los dilemas familiares con los que se está trabajando.

La postura que acaba de ser esbozada se mueve dentro del marco constructivista y socioconstruccionista considerando que cualquier descripción que realicemos sobre el otro es una descripción sobre nosotros como observadores mismos, puesto que aquello que llamamos realidad obedece a un acto interpretativo inmerso en el lenguaje. En el decir de Bateson (1979), el contexto en el que emerge un significado no puede aislarse del proceso interpretativo involucrado, toda vez que no somos observadores externos, pasivos y neutrales de la realidad, pues esta, desde el pensamiento postmoderno, es cuestionada y se plantea como una construcción en un proceso muy complejo que involucra elementos cognitivos, afectivos, intersubjetivos, sociales. En este orden de ideas, la supervisión es un proceso inquietante que reflexiona profundamente sobre nuestras posturas personales ante los casos de estudio y que desafía las verdades desde las que nos movemos, incorporando miradas disidentes y novedosas frente a la intervención con las familias. Como bien proponen Celis-Pacheco y RodríguezCebeiro (2017), "en ese ejercicio de humildad teórica, reconocemos que la observación del otro es necesaria, útil y, en muchas ocasiones, más pertinente que la nuestra" (p. 10).

Podría afirmarse que la supervisión es un espacio de conversación en el que emergen emociones, sentimientos y acciones desencadenadas por el vínculo y la cooperación. Se trata de un proceso riguroso y en movimiento dinámico entre la teoría y la práctica que, según plantea Minuchin (1998), amplía el escenario de la intervención poniendo en él mundos diversos y complejos, tal como unas gafas de aumento que permiten evidenciar otras realidades y ver distintos niveles de relaciones frente al dilema analizado. Cuando trabajamos con familias y escuchamos sus historias, es común que adoptemos sus puntos de vista y creamos que no hay salida alguna, tal como ellos piensan. La supervisión ayuda a que un árbol no nos tape el bosque (Rivett \& Buchmüller, 2018), amplía nuestra mirada, permitiéndonos vislumbrar nuevos caminos y comprensiones transformadoras del mismo dilema.

Este ejercicio comienza con la exposición del caso y el planteamiento de necesidades por parte del agente de intervención. En el proceso, identifica sus sentimientos, sus prejuicios, las hipótesis que subyacen a sus interacciones con las familias con las que trabaja, las creencias que están detrás de su relación con ellas, y analiza sus estilos de conexión con las familias que atiende. Busca identificar cómo juegan estos recursos en una dinámica de estudio y análisis reflexivo. Esto le exige descentrarse de su propio repertorio para incorporar y abrir nuevas puertas, dando acceso a comprensiones y posibilidades de los dilemas humanos dentro de los cuales navega cotidianamente. Al hacerlo, en la medida en que lleva a cabo su proceso, contribuye al desarrollo de quienes se unen en la dinámica de supervisión.

Se espera que sea el agente de intervención quien desarrolle la mayor cantidad de reflexiones, desatando nudos y proponiéndose nuevos propósitos de 
cara a la familia estudiada y a su desarrollo profesional, descubriendo nuevas fortalezas y complejizando su propia identidad dentro de la relación de ayuda. Por su parte, debido a que se trata de un ejercicio sistémico y complejo, los participantes enriquecerán sus miradas dando cuenta constantemente de sus prácticas y de sus referentes. Es esto lo que permite dar un giro a unos y otros, acordando nuevas maneras de acercarse a las familias con las que se trabaja, recordando que el norte es el bienestar de éstas y el confort de los equipos que trabajan con ellas. Significa que el enriquecimiento no va a ser sólo para el equipo que expone el caso de estudio sino para los distintos niveles de intervención en la supervisión, y también contundentemente, para las familias. Las nuevas comprensiones de los agentes de intervención y la ruptura de sellantes que, a veces, parecen atar los procesos serán caminos menos espinosos y rocosos por donde continuar desarrollando las conversaciones con las familias.

Estas nuevas comprensiones provienen de los sistemas de observación que se activan dentro de la supervisión, en tanto la conversación aporta distinciones y con ellas nuevas perspectivas sobre el caso a estudiar (Maturana \& Varela, 1986). El intercambio de puntos de vista es crucial para generar una nueva visión iluminadora, haciendo uso de todo tipo de recursos metafóricos, parábolas, comparaciones, explicaciones, etcétera (Von-Foerster, 1994). Se distinguen así el marco de referencia inicial del agente de intervención, el de las familias, y los nuevos mundos posibles, más satisfactorios, que las familias pueden llegar a visualizar y vivir.

\section{De las diferentes formas de abordar el ejercicio de la supervisión}

Para entretejer distintas formas de acción que conduzcan tanto a los equipos como a las familias hacia lugares más alentadores y seguros, la apertura a la construcción colaborativa implica la apertura de los escenarios frecuentemente incómodos y escasos de recursos en los que transcurre, pero, a la vez, validar diferentes formas de exponerse y enriquecer a partir de distintas miradas la acción de intervención con familias. Dependiendo del contexto en que se desarrolla el proceso y de la disponibilidad de recursos humanos de las instituciones involucradas, se acudirá a un supervisor profesional que acompañará el caso, sin embargo, muchas veces los agentes de intervención deberán construir su propia red de apoyo entre pares que funjan como supervisores entre sí. Es así como los escenarios de la supervisión pueden ser muy variados, pues ésta puede concertarse y llevarse a cabo detrás del espejo de la cámara de Gesell, en presencia directa durante sesiones en vivo, en una oficina destinada a la conversación sobre los casos, o puede suceder de forma espontánea mientras los colegas de la institución comparten un café o un paseo por el parque (Charles \& Nelson, 2019).

Es común y recomendable el uso de la cámara de Gesell o el estudio del video de una intervención con la familia, de preferencia la última realizada, ya que es de tener en cuenta que si un equipo pide el apoyo de otros es porque necesita en un contexto determinado: despegar, desatascar, desanudar, o dar un giro a una situación. Si no se pudiese contar con estos recursos, se recurre a la presentación 
verbal de una historia por parte del equipo o profesional que atiende el caso en cuestión.

Una alternativa es el sociodrama o escenificación de la situación familiar, o lo que es lo mismo, la representación del drama o guion que trae la familia, a manera de analogía teatral (Donoso-Castillo, 2007). Esto se puede realizar de dos maneras: a) Los agentes de intervención exponen el caso y se desarrolla la escena interpretando el rol de agente de intervención y de miembros de la familia, mientras los supervisores observan, luego de lo cual hacen preguntas o afirmaciones, y b) El agente de intervención expone el caso y se desarrolla la escena en la que éste y sus compañeros de equipo hacen el rol de los miembros de la familia, mientras los supervisores interpretan el rol de agentes de intervención. De cualquier forma, y si se cuenta con un equipo de varios participantes, pueden elegirse algunos observadores adicionales.

La supervisión puede darse durante una sesión con la familia en vivo y en directo, en la que participa el agente de intervención junto con el supervisor. Pueden emplearse técnicas diversas, entre las cuales se destaca el Equipo Reflexivo, expuesto por Tom Andersen (1994) e influenciado por la Escuela de Milán y por Lynn Hoffman. Se trata, entre otras cosas, de conversar sobre las conversaciones, esto es, de crear nuevas redes de interacción en las que el profesional dialoga con la familia mientras el supervisor escucha, o dialoga con el supervisor mientras la familia escucha, generando así nuevos significados, comprensiones, miradas, aprendizajes y desplazamientos de premisas.
En cualquier caso, en el estudio de las relaciones de la familia o sistema de intervención a supervisar, conviene hacer uso del genograma (Mc-Goldrick \& Gerson, 2003) y de otros recursos técnicos tales como los mapas de redes (Sluzki, 1996), que facilitan la representación de las relaciones dentro del sistema familiar, tanto entre ellos mismos (el equipo que expone el caso) como hacia el exterior. Al trabajar con estos recursos pueden identificarse y analizarse las conexiones entre los diferentes eventos de la historia de la familia, el momento actual de cada uno de los miembros del sistema y el momento del ciclo vital que atraviesa el sistema familiar.

Por otra parte, puede resultar útil conocer sobre el contexto social y los momentos relevantes de la historia familiar, identificando el significado que cada persona le otorga a los eventos vitales. No sobra mencionar, adicionalmente, que es importante encontrar posibles relaciones o resonancias entre los hitos vividos por las familias y las vivencias del agente de intervención o del supervisor. Es importante dedicar un rato a preguntarse acerca de ello, toda vez que este ejercicio toca la humanidad de los profesionales y "permite ocuparse de las emociones, hacerse cargo de los afectos, con lo que se accede a un mejor ejercicio del rol profesional desde el campo de la afectividad" (Puig, 2015, p. 60). Hay quienes buscan supervisar los casos que les generan más dificultad emocional que dificultad técnica, como hay quienes encuentran la resolución a las dificultades técnicas a partir de la exploración de las dificultades emocionales. 


\section{Acerca de los procedimientos de la supervisión}

Para que esta práctica llegue a buen término, el supervisor es responsable de una serie de tareas entre las cuales Sheehan (2017) destaca: construir la cohesión grupal del equipo que participa en el ejercicio de supervisión; aclarar los requerimientos y metas a seguir; proponer estructuras de aprendizaje y desarrollarlas; facilitar la evaluación de la supervisión, del proceso grupal y de los aprendizajes; su autocuidado y la supervisión de la supervisión, que deberá adelantar en otro escenario. Es importante, a su vez, que todos los participantes del proceso conozcan estas tareas, de modo que sean claros los límites propios de las responsabilidades del supervisor y que tanto las familias como los profesionales a cargo contribuyan al proceso.

Las conversaciones constituyen la materia prima de la supervisión. Entendemos la conversación como un acto más allá del lenguaje, en la cual éste es apenas uno de sus componentes, junto con la emocionalidad y la corporalidad, teniendo en cuenta que vivimos inmersos en redes de conversaciones que dan significado a lo que hacemos y cómo lo hacemos (Maturana \& Dávila, 2015). Cada persona y grupo tiene una forma particular de contar su historia, y es a través del diálogo que puede transmutar la mirada cerrada, obvia y simple sobre la realidad, teniendo en cuenta que "las narrativas no son historias fijas, sino que cada ser humano está en proceso de construir historias sobre los acontecimientos de su vida" (Hoyos-Botero, 2005, p. 163). La reflexión sobre nuestras maneras de conversar es la que amplía nuestra visión, cuestionando nuestras emociones y marcos de referencia de comprensión del caso y construyendo nuevas verdades consensuadas, ya que, al ver de otra manera, logramos pensar de otra forma (Hoffman, 1987).

Quienes participan en el ejercicio deben ser delicados con el uso del lenguaje, esforzándose por encontrar las palabras adecuadas para enriquecer y potenciar al equipo o al profesional que expone, en respeto y solidaridad, procurando estar atentos a sus propios prejuicios y alejarse de juicios incriminatorios o descalificantes hacia la familia o hacia quienes exponen su caso, movilizando aperturas y esperanzas de cara a los fines perseguidos. Veredictos, fórmulas, recetas, no caben en un proceso como éste. Por su parte, quienes exponen el caso al grupo, deberán revestirse de fortaleza y abrirse a la incorporación de nuevas miradas, mostrar gratitud hacia los esfuerzos hechos por los demás participantes para estudiar el dilema expuesto y contribuir a la creación de un clima en el que todos juegan del mismo lado.

Como se trata de una conversación entre profesionales que buscan fines orientados al fortalecimiento de las familias y de los equipos, las preguntas 0 afirmaciones deberán ser evidentemente enriquecedoras y circunscritas a ampliar y complejizar la mirada, a encontrar fortalezas, a evidenciar distinciones, a movilizar el sistema, a co-construir nuevas visiones de los dilemas, a potenciar el self del agente de intervención o equipo, a poner en tela de juicio construcciones reduccionistas, a incorporar curiosidades, caminos novedosos, y a comprender la situación en términos de inclusión y solidaridad. Es necesario insistir en que esto descarta las afirmaciones o preguntas 
que contengan juicios, consejos o fórmulas curativas.

Las conversaciones emergentes deberán cuestionar los relatos construidos por la familia o por el equipo de intervención, trayendo a la reflexión las emociones, temores, autorreferencias del profesional y lo que ocurre en la relación construida en el contexto del proceso de intervención. Los diálogos se verán especialmente vitalizados y enriquecidos por la indagación apreciativa (Cooperrider \& Whitney, 2005), desde la confianza en que tanto el profesional como el sistema familiar cuentan con las cualidades, recursos y potencialidades para superar sus dificultades. A su vez, el aporte de las preguntas circulares (Tomm, 1987) es fundamental, abriendo la puerta a nuevas miradas, distinciones y significados, al indagar sobre los diferentes puntos de vista de los participantes acerca de lo que los demás dicen, hacen y sienten. El uso de recursos narrativos es también útil para guiar las conversaciones, toda vez que las historias y metáforas posibilitan la expresión y comprensión de las situaciones, generando nuevos sentidos de reconocimiento y reencuadre (Cardona-Galeano \& Osorio-Sánchez, 2015). Veamos algunas preguntas que pueden servir como detonantes de este tipo de conversaciones, en los diferentes momentos del ejercicio de supervisión.

La supervisión suele iniciar con la definición del objetivo, o lo que es lo mismo, el pedido del equipo que presenta el caso. Para ello es útil preguntar al profesional que funge como agente de intervención: ¿Qué esperan de ese espacio? ¿Cuáles son las dificultades del proceso que los llevan a solicitar el apoyo? ¿Cómo se darían cuenta que la supervisión fue útil? ¿Con cuáles recursos personales cuentan para aprovechar este ejercicio de supervisión?

Después viene el momento central en el que se realizan conversaciones posibilitadoras, orientadas creativamente a realizar distinciones y a alcanzar mundos posibles de interpretación novedosos. Aquí destacan dos temas de conversación distintos, aunque estrechamente relacionados: la familia y la relación generada con ella. Respecto al primero, algunas preguntas enriquecedoras son: ¿cuál es el sueño que tiene esta familia, hacia dónde quieren ir como sistema?, ¿qué de lo que están haciendo deben cambiar o mantener para lograr ser la familia que quieren ser?, ¿qué pasos ha dado la familia hacia el cambio?, ¿hay soluciones intentadas por el sistema que se hayan vuelto parte del problema?, ¿qué versión tiene la familia de sí misma?, ¿cuáles relatos dominantes surgen en las narrativas de la familia?, ¿qué elementos surgen de relatos alternativos?, ¿hay en las historias, logros únicos que puedan resaltarse?, ¿cómo podrían hacerse visible esos logros únicos?, ¿qué elementos de la identidad de los miembros de la familia están siendo desafiados?, ¿cuáles son los principales recursos de la familia y de sus miembros?, ¿qué éxitos ha logrado la familia como sistema? En el pasado, ¿en qué momentos la familia se ha sentido verdaderamente orgullosa y feliz?, ¿hay mensajes paradójicos?, ¿de quién a quién? Si el síntoma fuera una metáfora con un mensaje para la familia, ¿qué estaría comunicando?

Por otra parte, respecto al segundo tema de conversación, las preguntas giran en torno a las emociones, experiencias y prejuicios del agente de intervención o 
equipo, así como sobre los desafíos que enfrenta como parte de la relación construida con la familia. Pueden proponerse interrogantes como: ¿qué cosas de su vida y/o de su rol como profesionales les obstaculizan dar un paso adicional con la familia en estos momentos?, ¿cuál es su mayor deseo y cuál su mayor temor respecto a esta familia?, ¿qué imágenes de esta familia les hacen sentirse más a gusto y cuáles les preocupan más?, ¿qué metáfora creen que permite ilustrar los dilemas que enfrenta esta familia?, ¿cuál ha sido la idea más novedosa y útil que ustedes como profesionales han tenido sobre el caso?, ¿qué recursos tienen ustedes como profesionales y seres humanos para acompañar a esta familia, y en qué situaciones los han evidenciado?

Como último momento de la supervisión, en el cierre del ejercicio se reconocen los aportes de los participantes y se precisan las nuevas conexiones que han surgido frente al caso; las preguntas que pueden abordarse incluyen: ¿qué cosa les gustaría decir a esta familia acerca de la conversación que hoy hemos tenido aquí?, ¿qué cosas definitivamente no compartirían a la familia, acerca de esta conversación?, ¿qué piensa que la familia necesita escuchar de ustedes, después de esta conversación?, ¿qué nueva hipótesis le gustaría desafiar?, ¿qué prejuicios propios intentarán auscultar, revisar, ampliar, modificar o mantener al margen? Si hoy no hubiéramos podido tener este espacio y ustedes tuvieran que trabajar con la familia, ¿qué hubieran hecho?, ¿qué nuevas conversaciones introducirán en su estilo de intervención personal?, ¿qué se llevan del ejercicio de supervisión? Se tratará de asegurar nuevos escalones de ejercitación y afinamiento de las acciones, identificar y potencializar los aciertos, propiciando que emerjan alternativas y posibilidades provocativas para todos. Un proceso así da lugar a la creatividad, por lo cual habrá que alejarse de senderos tortuosos y acercarse a la diversión, la exploración, la introducción de novedades y la experimentación de opciones.

\section{La supervisión como obligación ética}

La práctica supervisada es pertinente considerando que tal gestión apunta al robustecimiento de la intervención con las familias, pero, principalmente, se trata de una postura ética que va más allá de la mera incorporación técnica de conocimientos y del desarrollo de competencias. Atenderse, cuidarse, es también una necesidad de los agentes de intervención, que con frecuencia encuentra acogida en el acompañamiento de la red social que se construye entre los profesionales de los equipos que trabajan con familias. La supervisión, finalmente, se centra en la solidaridad de una red de apoyo a través de la construcción colectiva de análisis que discuten y desafían las potencialidades de todos, persiguiendo el bienestar laboral de los profesionales mientras se protege y cuida a las familias (Samson, Tanchak, Drolet, Fulton \& Kreitzer, 2019).

Una actitud curiosa, crítica e inquieta acerca de los procesos de cambio de los miembros del sistema familiar es fundamental, pero "se requiere sobre todo un cuestionamiento sobre la postura personal ante todos ellos y un afinamiento de las competencias para que la intervención sea en efecto transformadora de las experiencias familiares que constituyen los motivos de ayuda e intervención" (ICBFOIM, 2009, p. 94). La capacidad de quien 
trabaja con problemáticas familiares para analizar su estilo personal de relacionamiento con el caso, reflexionar sobre sus emociones y pulir sus destrezas de abordaje profesional, son aspectos que favorecen el alcance y efectividad de los procesos de acompañamiento (Isaza-Valencia \& Ocampo-Otálvaro, 2015). Es así cómo la reflexión sobre los propios marcos de comprensión de los dilemas familiares de parte del agente de intervención favorece el surgimiento de nuevas miradas acerca del caso y la comprensión de las relaciones familiares desde distintos lugares, que serán la llave con la que se abrirán cortinas para que puedan emerger los cambios deseados.

Desde la posición de Varela (2002), ampliar, cuestionar o defender los conocimientos personales constituye una postura ética toda vez que nuestras miradas, lejos de ser neutrales, dan sentido, se enfocan, hacen énfasis, distorsionan, no ven, constituyen en esencia solo una manera de observar. Se trata, entonces, de acercarnos a las situaciones de las familias desde una perspectiva de incertidumbre, lejos de la experticia tradicionalmente adjudicada a quienes quieren curar o arreglar problemas ajenos cual poseedores de la verdad que debe comunicarse y cumplirse; de abrirnos con humildad a reconocer nuestras limitaciones, valores, prejuicios y emocionalidad como seres humanos, de cuestionarlos identificando también nuestras fortalezas y aciertos, redundando en la potenciación de los propios recursos que poseemos como profesionales al incorporar nuevos sentidos de comprensión y modos de acción.

Se desprende de lo anterior que es indispensable incorporar en los mapas mentales de los agentes de intervención y sus supervisores el concepto ético que involucra a los otros como potencialmente capaces, llenos de recursos y fortalezas, considerando sus dilemas como vitales y humanos. Asimismo, la actitud sensible y cuidadosa será fundamental, caracterizada por la reflexión humilde sobre las propias limitaciones, prejuicios y emociones, reconociendo el ejercicio de supervisión como posibilidad de cambio orientada, también, al crecimiento personal y profesional de todos. Esto significa que el supervisor no debe asumirse como quien va a identificar y corregir los errores de los supervisados, sino como aquel acompañante que aportará miradas externas y preguntas que movilicen la reflexión conjunta sobre nuevos caminos de acción (Burck \& Daniel, 2018).

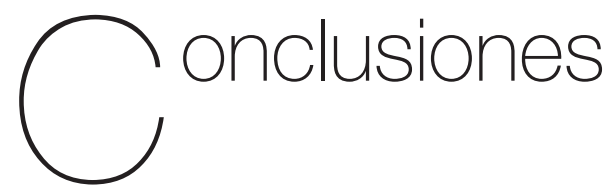

La conversación que proponemos realizar a manera de ejercicio de supervisión es sobre la existencia humana y la vida misma, haciendo más necesaria esta actitud ética de permanente autoobservación en el cuestionamiento de nuestras verdades de expertos. Siguiendo a Echeverría (2007), en este tipo de conversaciones no es posible una mirada fría y desinteresada que, por el contrario, al hablar y al escuchar nos enternecemos, nos conmovemos, nos entibiamos, toda vez que "la noción de verdad, siempre tan fría, no logra resistir ese aumento de la temperatura y se deshiela, convirtiéndose en algo muy diferente de lo que estábamos acostumbrados. De alguna manera, pareciera 
devenir menos absoluta, diríamos incluso menos verdadera" (p. 49).

Es por ello que la actitud del profesional que funge como supervisor requiere, a su vez, moverse con sensibilidad sobre las conversaciones que devienen en su ejercicio. Menciona Hernández-Córdoba (2007b) que es posible que el supervisor se descentre del rol de poder "puntuando la experticia solo como una forma de ver el mundo, sin desconocer las exigencias éticas de ofrecer a los consultantes el mejor proceso terapéutico posible y la participación que en ello tiene la experiencia previa del supervisor" (p.235). Hablamos, sin duda, de la realización de acciones humanas amorosas, solidarias, de vínculos que, si bien se desarrollan en el campo laboral, van más allá de ello pues involucran relaciones que tocan profundamente nuestro sentir, nuestro querer, nuestra humanidad, y en las que todos ganamos.

La práctica de la supervisión implica abanderarse de la inclusión que valora e incorpora los saberes de las familias, de los agentes de intervención y de los supervisores, para maravillarse de las capacidades de todos para sobreponerse a las adversidades, sorpresas o reveses que la vida les presenta, evidenciarlos y traerlos al escenario del cambio. Se trata de reconocer el poder transformador de la pregunta, la metáfora y la escucha, validando la potencialidad del acto conversacional en la construcción de realidades más amables para quienes, eventualmente, puedan estar paralizados ante cualquier obstáculo propio de su ser vulnerable. Consiste, finalmente, en la edificación de equipos de apoyo entre seres humanos diversos, cuyas relaciones se establecen de forma horizontal, validando los saberes de todos y llamando la atención sobre el trabajo en red como elemento fundamental de los procesos de transformación individual y colectiva.

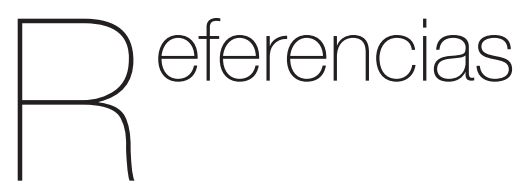

Andersen, T. (1994). El equipo reflexivo. Diálogos sobre los diálogos. Barcelona: Gedisa.

Bateson, G. (1979). Espíritu y naturaleza. Buenos Aires: Amorrortu.

Burck, C. \& Daniel, G. (2018). Mirrors and Reflections: Processes in Systemic Supervision. New York: Routledge.

Cardona-Galeano, I. L. \& Osorio-Sánchez, Y. L. (2015). Uso de la metáfora en terapia familiar. Aportes al enfoque narrativo. Revista Virtual Universidad Católica del Norte, 44, 15-35.

Celis-Pacheco, R. \& Rodríguez-Cebeiro, M. (2017). Constructivismo y Construccionismo social en psicoterapia. Bogotá: El Manual Moderno.

Charles, L. L. \& Nelson, T. S. (2019). Family Therapy Supervision in Extraordinary Settings. New York: Routledge.

Cooperrider, D. L. \& Whitney, D. (2005). A positive revolution in change. San Francisco: Berrett-Koehler Publishers.

Donoso-Castillo, A. (2007). La supervisión en terapia familiar sistémica: mirar y ver y verse 
y volver a mirar. Revista Trabajo Social, (6), 123-140.

Echeverría, R. (2007). Actos de Lenguaje Volumen l: La Escucha. Santiago, Chile: Comunicaciones Noreste Ltda.

Fruggeri, L. (2001). La supervisión desde diferentes niveles de análisis: entrenamiento y práctica reflexiva. Sistemas Familiares, 17(2), 88-100.

Hernández-Córdoba, A. (2007a). Trascender los dilemas del poder y del terapeuta como experto en la psicoterapia sistémica. Universitas Psychologica, 6(2), 285-293.

Hernández-Córdoba, A. (2007b). Supervisión de psicoterapeutas sistémicos: un crisol para devenir instrumentos de cambio. Diversitas. Perspectivas en Psicología, 3(2), 227-238.

Hoffman, L. (1987). Fundamentos de la terapia familiar. México: Fondo de Cultura Económica.

Hoyos-Botero, C. (2005). Aproximación a una psicología postmoderna: Una reflexión epistemológica. Informes Psicológicos, 7, 137-175. Recuperado de https://revistas.upb.edu.co/index. php/informespsicologicos/article/ view/1672/1616

ICBF-OIM. (2009). Lineamientos técnicos para la inclusión y atención de familias. Recuperado de https://repository.oim.org. co/bitstream/handle/20.500.11788/477/ COL-OIM\%200238.pdf?sequence=1

Isaza-Valencia, L. \& Ocampo-Otálvaro, L. E. (2015). Personalidad y competencias del terapeuta de niños, niñas y adolescentes que han vivido experiencias de abuso sexual, revisión teórica. Informes Psicológicos, 15(2), 93-110. Recuperado de https://revistas.upb.edu.co/index. php/informespsicologicos/article/ view/5687/5265

Maturana, H. \& Dávila, X. (2015). El árbol del vivir. Santiago, Chile: MVP editores.

Maturana, H. \& Varela, F. (1986). El árbol del conocimiento. Santiago, Chile: Ed. Universitaria.

Mc-Goldrick, M. \& Gerson, R. (2003). Genogramas en la Evaluación Familiar. Barcelona: Gedisa.

Minuchin, S. (1998). El arte de la terapia familiar. Barcelona: Paidós.

Puig, C. (2011). La supervisión en los equipos de Servicios Sociales: una oportunidad para la reflexión, el pensamiento y el cuidado de los profesionales. Cuadernos de Trabajo Social, 24, 123-133.

Puig, C. (2015). La supervisión en la acción social. Una oportunidad para el bienestar de los profesionales. Tarragona: Publicaciones URV. Recuperado de http:// llibres.urv.cat/index.php/purv/catalog/ download/211/247/485-1?inline=1

Rivett, M. \& Buchmüller, J. (2018). Family therapy skills and techniques in action. New York: Routledge.

Samson, P., Tanchak, S., Drolet, J., Fulton, A. \& Kreitzer, L. (2019). The Contribution of Clinical Supervision to Wellness in the Workplace: Implications for Social Work Field Education. Field Educator, 9(1), 1-24. Recuperado de http://www2.simmons. edu/ssw/fe/i/18-212.pdf 
Sheehan, J. (2017). Beginning the Supervisory Relationship within Family Therapy Training: Engaging Individuals, Groups and Settings. In A. Vetere \& J. Sheehan (eds.), Supervision of Family Therapy and Systemic Practice (pp. 3-26). Cham, Suiza: Springer.

Sluzki, C. (1996). La red social: Frontera de la práctica sistémica. Barcelona: Gedisa.

Tomm, K. (1987). Interventive interviewing: Part I. Strategizing as a fourth guideline for the therapist. Family Process, 26, 3-13.
Varela, F. (2002). El fenómeno de la vida. Santiago de Chile: Dolmen Ediciones.

Von-Foerster, H. (1994). Visión y conocimiento: disfunciones de segundo orden. En D. Fried Schnitman (comp.), Nuevos paradigmas, cultura y subjetividad (pp. 91-113). Buenos Aires: Paidós 